\title{
The Availability of Radiological Measurement of Tibial Torsion: Three-Dimensional Computed Tomography Reconstruction
}

\author{
Sang-yeop Shin, M.D., Chull Ho Yoon, M.D., Eun Shin Lee, M.D., Min-Kyun Oh, M.D., \\ A Ram Kim, M.D., Jong Moon Park, M.D., Jun-Hwa Shin, M.D., Hee Suk Shin, M.D. \\ Department of Rehabilitation Medicine and Institute of Health Sciences, \\ Gyeongsang National University Graduate School of Medicine, Jinju 660-702, Korea
}

\begin{abstract}
Objective To assess the intra-rater and inter-rater reliability for measuring tibial torsion measurements by a radiographic method using three-dimensional computed tomography reconstruction (3D-CT) and to compare the physical measures to those of 3D-CT.

Method The study included 33 children who presented with intoeing gait. Tibial torsion was measured by 3D-CT. Distal reference point was the bimalleolar axis. Proximal reference points were the transtibial axis and posterior condylar axis. Physical measurements included thigh-foot angle (TFA) and bimalleolar angle (BMA). 3D-CT measurement and physical measurement were performed twice at both lower extremities by each rater. The intra-rater and inter-rater reliability were calculated by intraclass correlation coefficiency (ICC). The relationship between radiological and physical examination was calculated by Spearman correlation coefficient.

Results The 3D-CT measures for tibial torsion were reliable within individual raters and between different raters. However, physical measures for tibial torsion were reliable within an individual rater but not reliable between raters. The 3D-CT measures by any proximal reference axis were more reliable within a rater and between raters than physical measurements. There was no significant impact introduced by the selection of the proximal reference axis. The correlation coefficiency between 3D-CT and physical measurement methods was low.

Conclusion Because the 3D-CT measurements for tibial torsion are more reliable than physical measurements, we recommend that accurate diagnosis of internal tibial torsion should be detected by using 3D-CT measurements. Also, considering the disadvantages of radiological measurements, physical measurement may be used for short term follow-up by same raters, as intra-rater reliability is relatively good.
\end{abstract}

Key Words Torsion abnormalities, Radiography, Tomography, Reproducibility of findings

Received June 1, 2011; Accepted July 16, 2011

Corresponding author: Hee Suk Shin

Department of Rehabilitation Medicine and Institute of Health Sciences, Gyeongsang National University Graduate School of Medicine, 90 Chilamdong, Jinju 660-702, Korea

Tel: +82-55-750-8255, Fax:+82-55-750-8255, E-mail: hsshin@nongae.gsnu.ac.kr

() This is an open-access article distributed under the terms of the Creative Commons Attribution Non-Commercial License (http://creativecommons. org/licenses/by-nc/3.0) which permits unrestricted noncommercial use, distribution, and reproduction in any medium, provided the original work is properly cited.

Copyright $\odot 2011$ by Korean Academy of Rehabilitation Medicine 


\section{INTRODUCTION}

The causes of intoeing gait include metatarsus adductus, internal tibial torsion, and internal femoral torsion, among which internal tibial torsion is known as the most common cause of intoeing gait. ${ }^{1}$ Tibial torsion is defined as the relationship between the axis of rotation of the knee and the transmalleolar axis. ${ }^{2}$ The indirect methods used to measure the value include physical examination of the patient's leg and radiological measurement methods such as simple X-ray, computed tomography (CT), nuclear magnetic resonance, ultrasonography, and fluoroscopy. ${ }^{3}$ During physical examination, the thigh foot angle (TFA) and bimalleolar angle (BMA) are commonly measured. ${ }^{2,4-6}$ However, the accuracy of physical examination has not been proven. ${ }^{7}$ On the contrary, the accuracy of CT was verified through a cadaver study, ${ }^{8}$ and thus most of the studies conducted until now have been to investigate the correlation between physical examination and CT. However, the two measurement methods showed a low correlation and the reliability and reproducibility of CT were superior, ${ }^{5,8}$ which was also verified in domestic studies. ${ }^{4,9}$ Other radiological methods including nuclear magnetic resonance and unltrasonography were suggested but these are also limited because the validity was acquired on the basis of the correlation with CT. Additionally, the recent development of medical imaging allows three dimensional computed tomography reconstruction (3D-CT). Studies were conducted on the reliability and accuracy of 3D-CT with respect to internal femoral torsion but not with respect to the tibial torsion.

Therefore, we proposed a new method to measure the tibial torsion angle using 3D-CT and evaluated the reliability of the method.

\section{MATERIALS AND METHODS}

\section{Subjects}

The subjects of this study were 33 infants who visited our hospital with the main complaint of intoeing gait and volunteered to participate in the study. Two physiatrists who learned the method to measure the tibial torsion angle measured the tibial torsion angle by performing physical examination and by using 3D-CT. Among the 33 infants, 8 were males, 25 were females, and the mean age
Table 1. Age and Sex Distribution of the Subjects

\begin{tabular}{lcc}
\hline \multicolumn{1}{r}{ Sex } & Number & Age \\
\hline Male & 8 & $5.38 \pm 4.59$ \\
Female & 25 & $6.40 \pm 3.65$ \\
Total & 33 & $6.18 \pm 3.81$ \\
\hline
\end{tabular}

Values are Mean \pm SD

was $6.18 \pm 3.81$ (Table 1).

\section{Methods}

Radiological method using 3D-CT: To measure the tibial torsion angle radiologically, the 3D-CT images were reconstructed. The distal reference axis was determined as the transmalleolar axis that connected the most protruding parts of the medial malleolus and lateral malleolus on the caudo-cranial view of 3D-CT. The proximal reference axis was determined as the following two axes on the cranio-caudal view: the transtibial axis that divides the tibial head into two and passes through the central region; and the posterior condylar axis that passes through the two most posterior joints on the tibial plateau. The angle between the distal reference axis and the proximal reference axis was measured as the tibial torsion angle (Fig. 1).

Two raters measured the left and right sides, having the transtibial axis and posterior condylar axis as the proximal reference axes (first session) and then the individual raters repeated the measurement in the order of patients different from the previous one, making the measurements of the first session and measurements by the other rater unknown (second session).

\section{Clinical measurement}

TFA measurement ${ }^{2,4,5}$ : The patient was positioned prone, the knee flexed to $90^{\circ}$, and ankle positioned in neutral dorsiflexion/plantarflexion. The point where the heel is divided into two and the point where the forefoot is divided into two were marked. Then, the angle between the lines that connect these two points and the line that divides the thigh was measured on the horizontal plane using a goniometer.

BMA measurement $t^{6}$ : The central points of the medial malleolus and lateral malleolus were marked with a pen. The patient was asked to be in the supine position, stretch the knee joint, and keep the feet in the neutral position. Then, the angle was measured by placing the two arms of 


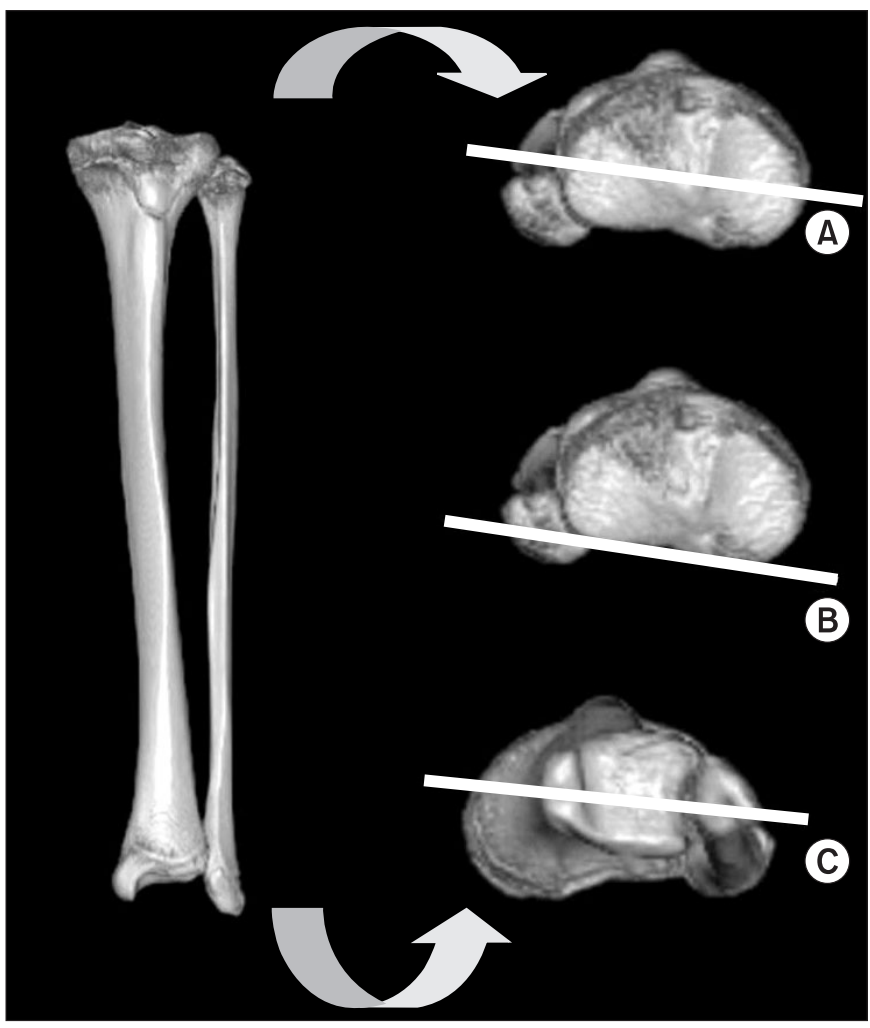

Fig. 1. Reference axes of 3-Dimensional CT reconstruction measurement. (A) Proximal transtibial axis, (B) Proximal posterior condylar axis, (C) Distal bimalleolar axis. Tibial rotation is the angle between the proximal and distal axis.

a vertical goniometer at the central points between the medial malleolus and lateral malleolus (Fig. 2).

Two raters measured the left and right sides (first session) and then the individual raters repeated the measurements at the next follow up, making the measurements of the first session and the measurements by the other rater unknown (second session).

\section{Statistical processing}

The SPSS version 19.0 was used for statistical analysis. The inter-rater reliability and the intra-rater reliability of the tibial torsion angle measurement method were analyzed using the intraclass correlation coefficient (ICC). The correlation between the physical measurement and the 3D-CT measurement was analyzed using Pearson's correlation coefficient.

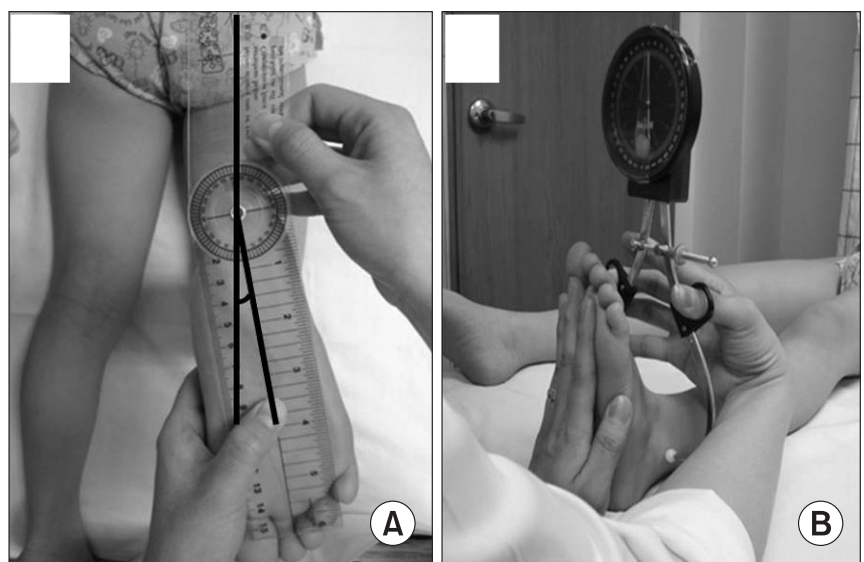

Fig. 2. Thigh foot angle which measures angle between longitudinal axis of the thigh and longitudinal axis of the foot (A) and bimalleolar angle measured by vertical goniometer at the center between medial and lateral malleolus (B), are shown.

\section{RESULTS}

The descriptive statistics of the tibial torsion angle measured using $3 \mathrm{D}-\mathrm{CT}$

In the measurement performed by Rater $\mathrm{A}$, the mean and standard deviation of the tibial torsion angle were $12.71 \pm 9.39^{\circ}$ and $12.73 \pm 8.73^{\circ}$ in the first and the second sessions, respectively, with reference to the transtibial axis as the proximal axis. The mean values were $15.72 \pm 9.26^{\circ}$ and $15.81 \pm 8.99^{\circ}$ in the first and the second sessions, respectively, with reference to the posterior condylar axis as the proximal axis. In the measurement performed by Rater B, the mean and standard deviation of the tibial torsion angle were $12.53 \pm 8.07^{\circ}$ and $12.58 \pm 7.50^{\circ}$ in the first and the second sessions, respectively, with reference to the transtibial axis as the proximal axis. The mean values were $14.76 \pm 7.90^{\circ}$ and $15.16 \pm 7.33^{\circ}$ in the first and the second sessions, respectively, with reference to the posterior condylar axis as the proximal axis (Table 2).

The inter-rater reliability and intra-rater reliability of the tibial torsion angle measurement method using 3D-CT

The correlation coefficient of the tibial torsion angle with reference to the transtibial axis between the first and the second session was 0.75 for Rater A and 0.87 for Rater $B$. In that case, the correlation coefficient between Raters $\mathrm{A}$ and $\mathrm{B}$ was 0.69 . The correlation coefficient of the tibial torsion angle with reference to the posterior condylar 
Table 2. Mean and Standard Deviation Values of Tibial Torsion by Measurements using 3 Dimensional CT Imaging and Physical Methods

\begin{tabular}{lcrc}
\hline & Session & Rater A & Rater B \\
\hline CT-trans & 1st & $12.71 \pm 9.39$ & $12.53 \pm 8.07$ \\
& 2nd & $12.73 \pm 8.73$ & $12.58 \pm 7.50$ \\
CT-posterior & 1st & $15.72 \pm 9.26$ & $14.76 \pm 7.90$ \\
& 2nd & $15.81 \pm 8.99$ & $15.16 \pm 7.33$ \\
TFA & 1st & $2.70 \pm 7.14$ & $5.06 \pm 7.44$ \\
& 2nd & $1.85 \pm 6.63$ & $4.94 \pm 6.19$ \\
BMA & 1st & $7.65 \pm 7.29$ & $7.89 \pm 10.48$ \\
& 2nd & $6.95 \pm 8.85$ & $6.03 \pm 7.62$ \\
\hline
\end{tabular}

Values are Mean \pm SD

CT-trans: 3-Dimensional computed tomography referenced transtibial axis as proximal axis, CT-posterior: 3-Dimensional computed tomography referenced posterior condylar axis as proximal axis, TFA: Thigh foot angle, BMA: Bimalleolar angle

Table 3. Intra-rater Reliabilities of Tibial Torsion Measures between First Session and Second Session

\begin{tabular}{llcc}
\hline & & Rater A & Rater B \\
\hline 3D-CT & CT-trans & 0.75 & 0.87 \\
measures & & $(0.63-0.84)$ & $(0.79-0.92)$ \\
& CT-posterior & 0.77 & 0.86 \\
& & $(0.65-0.85)$ & $(0.78-0.91)$ \\
Physical & TFA & 0.70 & 0.70 \\
measures & & $(0.55-0.85)$ & $(0.56-0.81)$ \\
& BMA & 0.74 & 0.83 \\
& & $(0.61-0.85)$ & $(0.74-0.89)$ \\
\hline
\end{tabular}

Values are Intraclass Correlation Coefficient (95\% Confidence Interval)

CT-trans: 3-Dimensional computed tomography referenced transtibial axis as proximal axis, CT-posterior: 3 -Dimensional computed tomography referenced posterior condylar axis as proximal axis, TFA: Thigh foot angle, BMA: Bimalleolar angle

axis between the first and the second session was 0.77 for Rater A and 0.86 for Rater B. In that case, the correlation coefficient between Raters A and B was 0.71 (Table 3, 4).

\section{The descriptive statistics of the tibial torsion angle} measured by physical examination by the individual raters

In the measurement performed by Rater $\mathrm{A}$, the mean and standard deviation of the TFA value were $2.70 \pm 7.14^{\circ}$
Table 4. Inter-rater Reliabilities of Tibial Torsion Measurements between Rater A and Rater B

\begin{tabular}{llcc}
\hline & & $\begin{array}{c}\text { Intraclass } \\
\text { correlation } \\
\text { coefficient }\end{array}$ & $\begin{array}{c}\mathbf{9 5 \%} \\
\text { confidence } \\
\text { interval }\end{array}$ \\
\hline 3D-CT & CT-trans & 0.69 & $0.59-0.77$ \\
measures & CT-posterior & 0.71 & $0.61-0.78$ \\
Physical & TFA & 0.25 & $0.08-0.40$ \\
measures & BMA & 0.02 & $-0.14-0.19$ \\
\hline
\end{tabular}

CT-trans: 3-Dimensional computed tomography referenced transtibial axis as proximal axis, CT-posterior: 3-Dimensional computed tomography referenced posterior condylar axis as proximal axis, TFA: Thigh foot angle, BMA: Bimalleolar angle

Table 5. Pearson Correlation Coefficients of Tibial Torsion Measurements between Physical Examination and 3-Dimensional CT

\begin{tabular}{llc}
\hline $\begin{array}{c}\text { Physical } \\
\text { examination }\end{array}$ & $\begin{array}{c}\text { 3-Dimensional } \\
\text { CT }\end{array}$ & $\begin{array}{c}\text { Correlation } \\
\text { coefficient (r) }\end{array}$ \\
\hline TFA & CT-trans & 0.11 \\
& CT-posterior & $0.16^{*}$ \\
BMA & CT-trans & $0.16^{*}$ \\
& CT-posterior & $0.19^{*}$ \\
\hline
\end{tabular}

CT-trans: 3-Dimensional computed tomography referenced transtibial axis as proximal axis, CT-posterior: 3 -Dimensional computed tomography referenced posterior condylar axis as proximal axis, TFA: Thigh foot angle, BMA: Bimalleolar angle ${ }^{*} \mathrm{p}<0.001$

and $1.85 \pm 6.63^{\circ}$ in the first and the second sessions, respectively. The mean BMA values were $7.65 \pm 7.29^{\circ}$ and $6.95 \pm 8.85^{\circ}$ in the first and the second sessions, respectively. In the measurement performed by Rater B, the mean and standard deviation of the TFA value were $5.06 \pm 7.44^{\circ}$ and $4.94 \pm 6.19^{\circ}$ in the first and the second sessions, respectively. The mean BMA values were $7.89 \pm 10.48^{\circ}$ and $6.03 \pm 7.62^{\circ}$ in the first and the second sessions, respectively (Table 2 ).

The inter-rater reliability and intra-rater reliability of the tibial torsion angle measurement method using physical examination

The correlation coefficients of the TFA and BMA values between the first and the second sessions were 0.70 and 0.74 , respectively, by Rater A, and those of Rater B were 
A

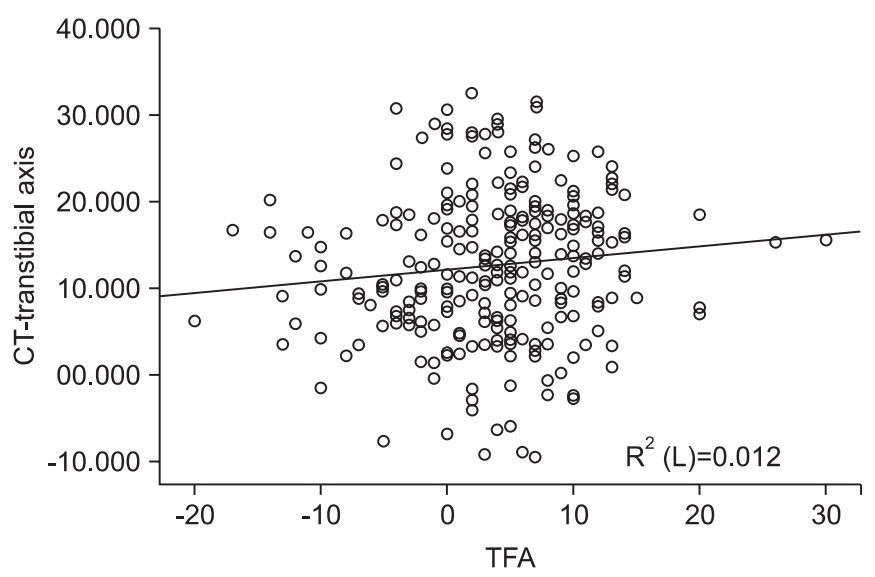

C

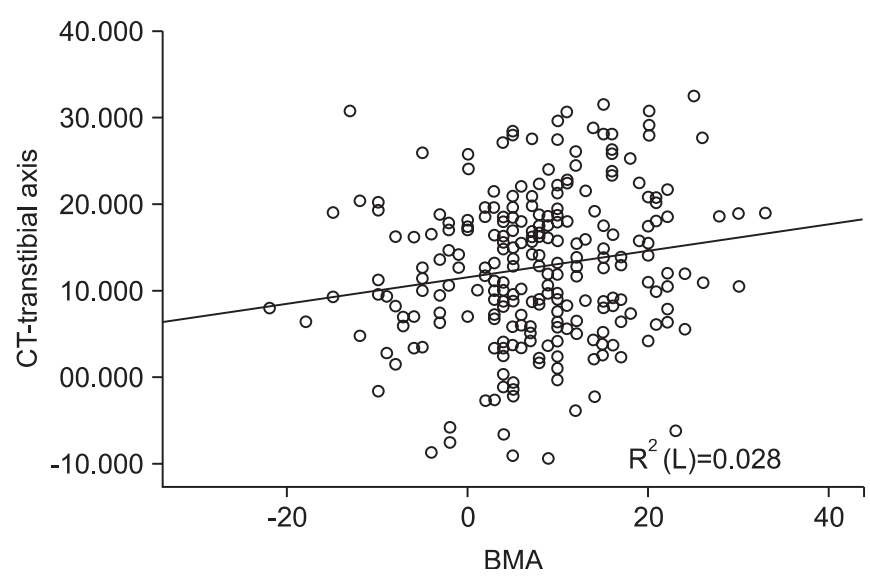

B

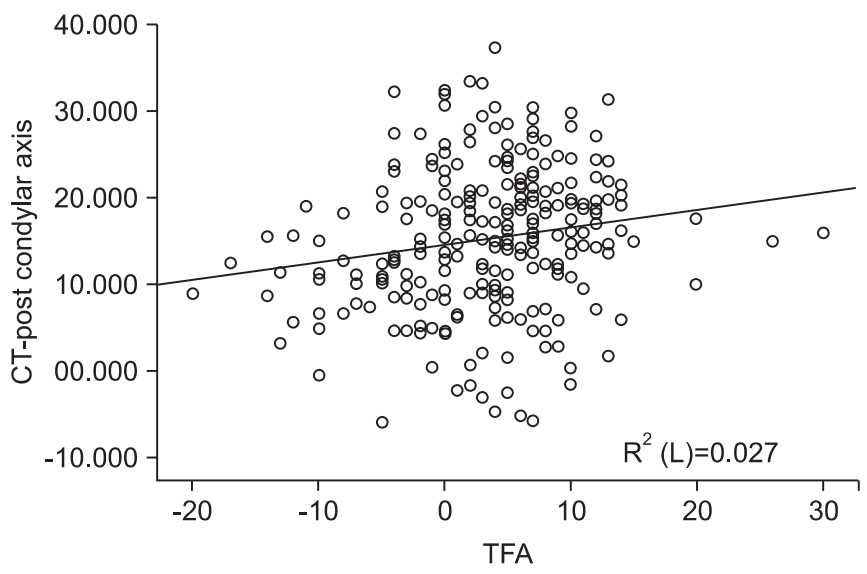

D

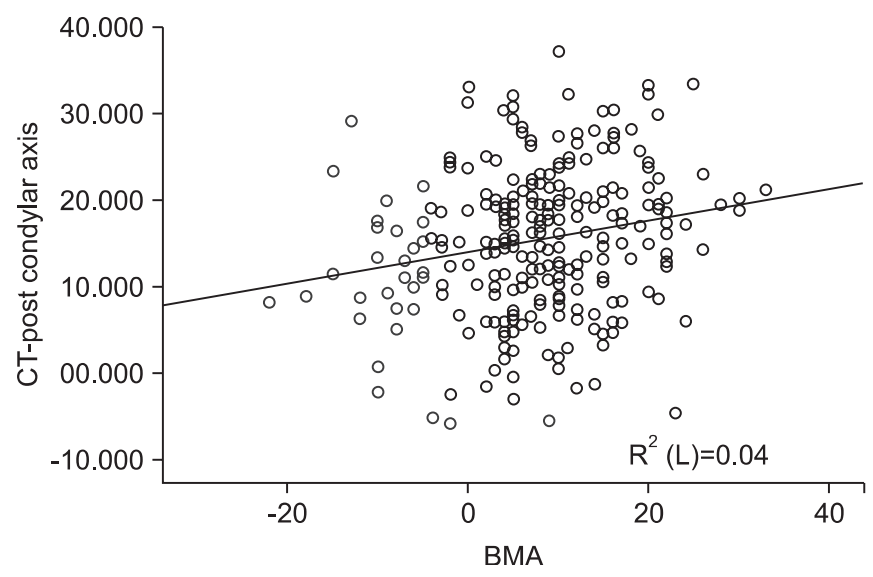

Fig. 3. Relationship between 3D-CT measurment referenced by transtibial axis as proximal axis and physical examination (TFA and BMA) (A and C) Relationship between 3D-CT measurment referenced by posterior condylar axis as proximal axis and physical examination (TFA and BMA) (B and D).

0.70 and 0.83 , respectively. The correlation coefficient of the TFA and BMA values between Raters A and B were 0.25 and 0.02 (Table 3, 4).

The correlation between the tibial torsion angles measured by physical examination and 3D-CT

The correlation coefficient between physical examination and 3D-CT measurement method was in a range between 0.11-0.19, indicating a low level of correlation (Table 5, Fig. 3).

\section{DISCUSSION}

Infants may show intoeing gait normally during the growth and development process, which is improved naturally in most cases. ${ }^{2}$ If continued, however, it may result in cosmetic, functional, and psychologic problems, and if serious, may cause degenerative arthritis or chondromalacia patella. ${ }^{10}$ Thus, treatment of tibial torsion, the most common cause of intoeing gait, is very important and should be based on accurate diagnosis. Various methods of physical examination and radiologic measurement have been applied to measure the tibial torsion angle..$^{4-6,8,9,11-13}$

Among these methods, physical examination is most commonly used in clinics. However, in a cadaver study that investigated the accuracy of physical examination, significant difference was verified with direct measurement. ${ }^{7}$ Studies on the correlation with CT also showed that the correlation was not very high. For example, Lee et al. ${ }^{9}$ found a mild correlation coefficient between physical examination and the two-dimensional computed tomography (2D-CT) in 18 children with cerebral palsy. Stuberg et al. ${ }^{5}$ also verified the significant 
difference between the two measurement methods in 17 normal subjects.

Because of the limitations of physical examination, studies on radiological evaluation methods are still conducted. For example, 2D-CT is widely used as the method to measure the tibial torsion angle because a relatively high accuracy was verified in cadaver studies. ${ }^{8,13}$ Although the recent development of medical imaging allows 3D-CT, the reliability and accuracy of 3D-CT with respect to the tibial torsion angle measurement have not been investigated.

We assumed that the conventional two-dimensional measurement methods had a fundamental limitation in measuring the tibial torsion, because the tibia is a three-dimensional structure. Additionally, the excellent accuracy of 3D-CT for internal femoral torsion was verified already. ${ }^{14,15}$ Thus, we presumed that the application of 3D-CT to the measurement of internal tibial torsion would be more accurate.

Therefore, we conducted this study to suggest the application of 3D-CT, not 2D-CT, as a method to measure the tibial torsion angle. Because the reliability and accuracy should be high for a measurement method to be widely used, this study primarily focused on whether the reliability of the method could be verified clinically. The results showed a high intra-rater reliability and interrater reliability of the $3 \mathrm{D}-\mathrm{CT}$ measurement method, indicating that it can be a useful tool for diagnosis and follow-up. On the contrary, physical examination of tibial torsion showed a low level of inter-rater reliability even though the intra-rater reliability was high. In other words, physical examination cannot provide objective information because the measurement result is greatly dependent upon the raters and the measurement methods, except for the continuous follow-up of the same infant patient by the same rater.

Moreover, the choice of the reference axis should be taken into account when applying the 3D-CT measurement method. To confirm the argument, Eckhoff and Johnson ${ }^{3}$ conducted a cadaver study comparing the 3D-CT measurement values of the cases where the transtibial axis, posterior condylar axis, and anterior condylar axis were chosen as the proximal reference axis The transtibial axis was defined as the bisector of the two lines that join the most anterior and posterior points on each half of the tibial plateau. The posterior condylar axis was defined as the line joining the two most posterior joints on the tibial plateau, which is the same as that of our study. The anterior condylar axis was defined as the line joining the most anterior points of each condyle or compartment of the tibial plateau. The comparison showed that the choice of proximal reference axis did not have a significant effect. Also, in our study, the results were compared for the cases where the transtibial axis and the posterior condylar axis were chosen, as they are chosen most widely as the proximal reference axes. ${ }^{3,8,13}$ The result showed that there was not a great difference in the reliability depending on the choice of proximal reference axis and the relation coefficient between the methods was as high as $0.86-0.93$, indicating that the result was consistent with that of the previous studies.

In summary, the measurement of the tibial torsion angle based on 3D-CT is a very useful method because it has a high reliability and is not greatly dependent on the choice of the reference axis. However, the limitations of the method should not be overlooked. For example, CT may be of limited value in children because of the incomplete ossification and it has the disadvantages of radiological exposure and high cost. ${ }^{11,12}$

In conclusion, considering the advantages and limitation of the methods to measure the tibial torsion angle, we suggest that the 3D-CT method should be used for accurate diagnosis of internal tibial torsion, whereas physical examination by the same rater should be used for the follow-up in a short period of time for same patient. However, it is inappropriate to assess the progression by comparing the two values measured by different methods because the correlation between the two measurement methods is low.

Moreover, it may be needed to verify the accuracy of the 3D-CT method by means of direct measurement, which is impossible in clinical settings. Thus, a future study should be focused on the assessment of the accuracy of the 3D-CT method to measure the tibial torsion angle through direct measurement in cadaver study. In addition, it is necessary to present the normal tibial torsion angle values among normal infants measured by the 3D-CT method and clarify the standards for the diagnosis of internal tibial torsion. 


\section{CONCLUSION}

The 3D-CT method to measure tibial torsion angle had higher intra-rater reliability $(0.75-0.87)$ and inter-rater reliability (0.69-0.87) than those of physical examination. The 3D-CT method also has the advantage that the result is not dependent on the choice of the proximal reference axis. Therefore, the 3D-CT method can be useful for the diagnosis and follow-up of the infants who have intoeing gait.

\section{REFERENCES}

1. Kumar SJ, MacEwen GD. Torsional abnormalities in children's lower extremities. Orthop Clin North Am 1982; 13: 629-639

2. Staheli LT, Corbett M, Wyss C, King H. Lower-ex tremity rotational problems in children. Normal values to guide management. J Bone Joint Surg Am 1985; $67: 39-47$

3. Eckhoff DG, Johnson KK. Three-dimensional computed tomography reconstruction of tibial torsion. Clin Orthop Relat Res 1994; 302: 42-46

4. Lee SY, Choi KS, Jeung IS, Lee JE, Yang SM, Lee SM. Physical examination and computed tomography in children with toe-in gait. J Korean Acad Rehab Med 2011; 35: 61-66

5. Stuberg W, Temme J, Kaplan P, Clarke A, Fuchs R. Measurement of tibial torsion and thigh-foot angle using goniometry and computed tomography. Clin Orthop Relat Res 1991; 272: 208-212

6. Valmassy R, Stanton B. Tibial torsion. Normal values in children. J Am Podiatr Med Assoc 1989; 79: 432-435

7. Milner CE, Soames RW. A comparison of four in vivo methods of measuring tibial torsion. J Anat 1998; 193: 139-144

8. Jakob RP, Haertel M, Stussi E. Tibial torsion calculated by computerised tomography and compared to other methods of measurement. J Bone Joint Surg Br 1980; 62: $238-242$

9. Lee SH, Chung CY, Park MS, Choi IH, Cho TJ. Tibial torsion in cerebral palsy: validity and reliability of measurement. Clin Orthop Relat Res 2009; 467: 20982104

10. Winter WG Jr, Lafferty JF. The skiing sequelae of tibial torsion. Orthop Clin North Am 1976; 7: 231-240

11. Clementz BG. Assessment of tibial torsion and rotational deformity with a new fluoroscopic technique. Clin Orthop Relat Res 1989; 245: 199-209

12. Guven M, Akman B, Unay K, Ozturan EK, Cakici H, Eren A. A new radiographic measurement method for evaluation of tibial torsion: a pilot study in adults. Clin Orthop Relat Res 2009; 467: 1807-1812

13. Jend HH, Heller M, Dallek M, Schoettle H. Measurement of tibial torsion by computer tomography. Acta Radiol Diagn (Stockh) 1981; 22: 271-276

14. Abel MF, Sutherland DH, Wenger DR, Mubarak SJ. Evaluation of CT scans and 3-D reformatted images for quantitative assessment of the hip. J Pediatr Orthop 1994; 14: 48-53

15. Kim SI, Park SB, Lee KM. Measurement of femoral anteversion using 3 dimensional imaging. J Korean Acad Rehab Med 1994; 18: 495-499 